\title{
Is there anyone out there?
}

\section{Washington}

A NEW search for extraterrestrial intelligence was launched last week at Harvard. Massachusetts. A multi-million channel receiver operated by the Planetary Society will search 8.4 million radio channels for possible signals from an alien civilization. 64 times more than the society's existing Project Sentinel. Construction of the new receiver, called META (for Megachannel Extraterrestrial Array) was financed by a $£ 100.000$ gift from Steven Spielberg. director of "ET", the blockbuster movie about the adventures of an alien stranded on Earth.

The antenna to be used by META is an 84-foot diameter dish at Harvard University's Oak Ridge Observatory which was due to be dismantled until the Planetary Society stepped in. The META receiver was designed and built by Paul Horowitz of Harvard University, and has been constructed for a knockdown price because it searches only selected "magic frequencies" - frequencies corresponding to emission frequencies of the most abundant atoms or radicals in the Universe. According to the argument, an alien civilization seeking to broadcast its presence might choose a "magic frequency" to send its messages. META will look initially for ultra-narrow bandwidth signals (using a

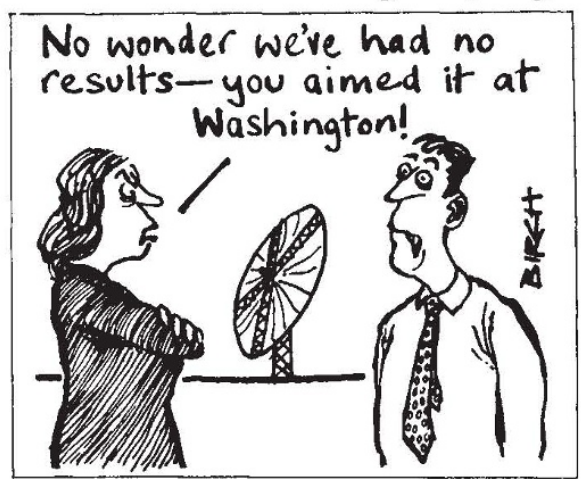

$0.04 \mathrm{~Hz}$ channel width) in the noisy 1,420 $\mathrm{MHz}$ region, corresponding to a major emission frequency of hydrogen.

Supporters of the "magic frequency" strategy argue further that an intelligent alien might correct the frequency of its broadcast for its own motion relative either to the Galaxy or to the Universe as a whole, so that a similarly intelligent listener, by compensating for its motion, could eliminate Doppler shift. The Earth's motion relative to the rest frame of the Universe and of our Galaxy is now known, and META will be the first receiver to search the $1,420 \mathrm{MHz}$ hydrogen band in these rest frames. A major emission frequency of the hydroxyl radical at 1,700 $\mathrm{MHz}$ might also be examined later.

Spielberg's gift was made on the condition that the Planetary Society continue to support operation of META for several years, which it plans to do from donations by members. Other searches for extraterrestrial intelligence are under way in the Soviet Union and elsewhere. Ohio State University's "Bigear" radio telescope. which supports one search, has for the past two years been under threat of demolition in order to make way for a golf course; last month the threat was averted. however, and the telescope now looks set to continue operations for at least another 10 years. And despite severe budget restrictions, the National Aeronautics and Space Administration (NASA) has been able to spend around $\$ 1.5$ million a year building a receiver that. when it comes into operation in 1988 , will search all frequencies that are reasonable for interstellar communication, from 1 to $10 \mathrm{GHz}$. But NASA's search, unlike that of Horowitz's instrument, will look for pulsed signals and examine individual nearby Sun-like stars.

Tim Beardsley

\section{Primate research}

\section{NIH withdraws support}

\section{Washington}

THE University of Pennsylvania last week banned primate research and reprimanded Thomas Gennarelli and Thomas Langfitt of the head injury research laboratory, but rejected allegations of cruel and inhumane treatment of animals. Representatives of People for Ethical Treatment of Animals, who last year stole researchers' videotapes, called the university's decision "most responsible". In studies that were carried out in collaboration with the University of Glasgow, in Scotland, the laboratory has been using primates to model the effects of brain damage in humans. Financial support for the research has now been withdrawn by the National Institutes of Health (NIH) pending a full enquiry, and the Department of Agriculture (DoA) is suing the laboratory for violation of the Animal Welfare Act.

The university has decided that $\mathrm{NIH}$ guidelines for animal research were not met in the supervision and training of staff or in sanitation and postoperative care of animals. These findings are considered "particularly troublesome" in view of worries already expressed by the university's animal care committee as long ago as 1982. Research will not be resumed until the committee, which is being reconstituted according to new public health guidelines, NIH and DoA, are satisfied.

Gennarelli and Langfitt said last week that all research programmes at the laboratory have been jeopardized as a result of the ban on primate projects, which represent less than a quarter of the laboratory's work (the rest is computer simulation and modelling). Gennarelli and Langfitt acknowledge some "minor infractions" but refuse to abandon the hope of resuming their work on injuries to primates. Although the university has reaffirmed its "strong support for the use of animals in biomedical research", Gennarelli and Langfitt think that permission to continue their "controversial" research will be withheld "for a long time" because of public protests.

Maxine Clarke

\section{US “big science” looks abroad}

\section{Washington}

THE US National Research Council (NRC) is contemplating launching a major study on whether increased international collaboration on "big science" would be a desirable means of increasing funds for major science and technology facilities. A blue-ribbon panel chaired by Frederick Seitz, a past president of the National Academy of Sciences, and Ralph Gomory, of IBM, spent two days last week discussing the question and will shortly make a recommendation for a formal study to NRC's Commission of Physical Sciences, Mathematics and Resources.

The study was inspired by growing concern that, with attempts to limit the national budget deficit a priority for Congress and the spiralling cost of the large facilities now required by physicists, some planned projects might face long delays unless other sources of funds are found. The proposed Superconducting Supercollider, which would probably cost more than
$\$ 3,000$ million, is an obvious case in point. Although international partners are unlikely at this stage to be interested in sharing construction costs, they might be willing to supply instruments for the collider, which may themselves cost hundreds of millions of dollars, according to NRC's Herbert Freidman.

Space research is another area with sig. nificant potential for international collaboration, according to Freidman; the planned International Solar Terrestrial Physics Program might become a model for future collaboration. Cooperation over the planned space station and space-based interferometry are also being considered, as are areas that might be included in the study.

The proposed study has yet to be formally approved by several layers of NRC bureaucracy, which could take months. After that, the search will be on for agencies willing to put up the money needed to pay for it.
Tim Beardsley 